\section{Rôle de la thrombospondine- 1 dans le développement des maladies rénales}

Naïke Bigé, Jean-Jacques Boffa, Fanny Lepeytre, Nasim Shweke

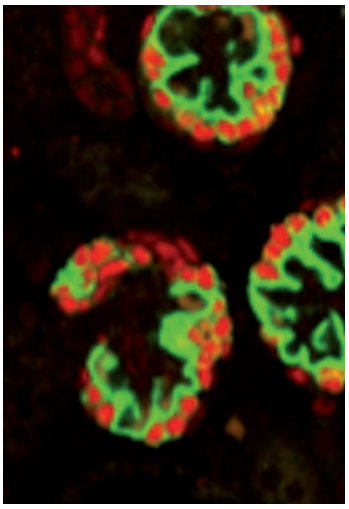

Service néphrologie et dialyse, Inserm UMRS 702, hôpital Tenon, 4, rue de la Chine, 75020 Paris, France.

jean-jacques.boffa@tnn.aphp.fr effet délétère sur la réparation du tissu rénal en favorisant le développement de la fibrose, la raréfaction capillaire et le recrutement des cellules inflammatoires. Ainsi, la TSP- 1 représente une cible thérapeutique potentielle pour la prise en charge des maladies rénales chroniques. <

La thrombospondine-1 (TSP-1) est la première protéine de la famille des thrombospondines (TSP) qui a été décrite, et elle est la plus étudiée. Elle interagit avec de nombreux partenaires et possède une activité anti-angiogénique, pro-apoptotique et immunomodulatrice. Chez l'adulte, l'expression de la TSP-1 se limite aux tissus inflammatoires ou en cours de remodelage; elle y régule la structure de la matrice extracellulaire, ainsi que le phénotype, l'activation et la prolifération cellulaires. Dans cette revue, nous nous intéresserons à son rôle dans la physiopathologie des maladies rénales.

\section{Structure de la thrombospondine-1}

La famille des TSP comporte cinq membres répartis en deux groupes. Le groupe A réunit la TSP-1 et la TSP-2 qui

Vignette (Photo (C) Inserm - Andreas Schedl) sont des glycoprotéines homotrimériques de structure homologue. Les TSP-3 à -5 , qui constituent le groupe $B$, sont des homopentamères. La TSP-1, glycoprotéine de 450 kDa identifiée par une analyse du contenu des granules $\alpha$ des plaquettes stimulées par la thrombine, a été la première décrite en 1971 .

Son domaine amino-terminal, constitué d'environ 200 acides aminés, se lie à l'héparine, aux protéoglycanes et aux intégrines $\alpha 3 \beta 1, \alpha 4 \beta 1, \alpha 5 \beta 1$ et $\alpha 6 \beta 1$ (Figure 1). II assure aussi sa clairance par le récepteur des lipoprotéines de faible densité (LRP). II existe ensuite un domaine d'oligomérisation, puis un domaine procollagénique identique au domaine de type $C \mathrm{du}$ facteur von Willebrand (vWF) qui participe à l'activité anti-angiogénique. Puis intervient le domaine TSRl (domaine de répétition de type 1) qui est caractérisé par trois répétitions du motif TSPc et qui contient les deux séquences indispensables à l'activation du TGF- $\beta$ (transforming growth factor- $\beta$ ). Ce domaine participe aussi à l'activité anti-angiogénique en interagissant avec les intégrines $\beta$ l et le CD36. La partie carboxy-terminale, appelée domaine de signature, hautement conservée au sein de la famille des TSP, comporte des domaines EGF (epidermal growth factor)-like (TSR2) répétés trois fois, suivis de 13 séquences de fixation au calcium. Le domaine globulaire carboxy-terminal lectine-like permet la fixation au récepteur CD47 ou IAP (integrin associated protein) (Figure 1) [1, 2].

\section{Fonctions de la thrombospondine-1}

\section{Activation du TGF- $\beta$}

La TSP-l est un activateur endogène du TGF- $\beta$ qui est un facteur profibrosant majeur. Elle forme un complexe trimoléculaire avec 


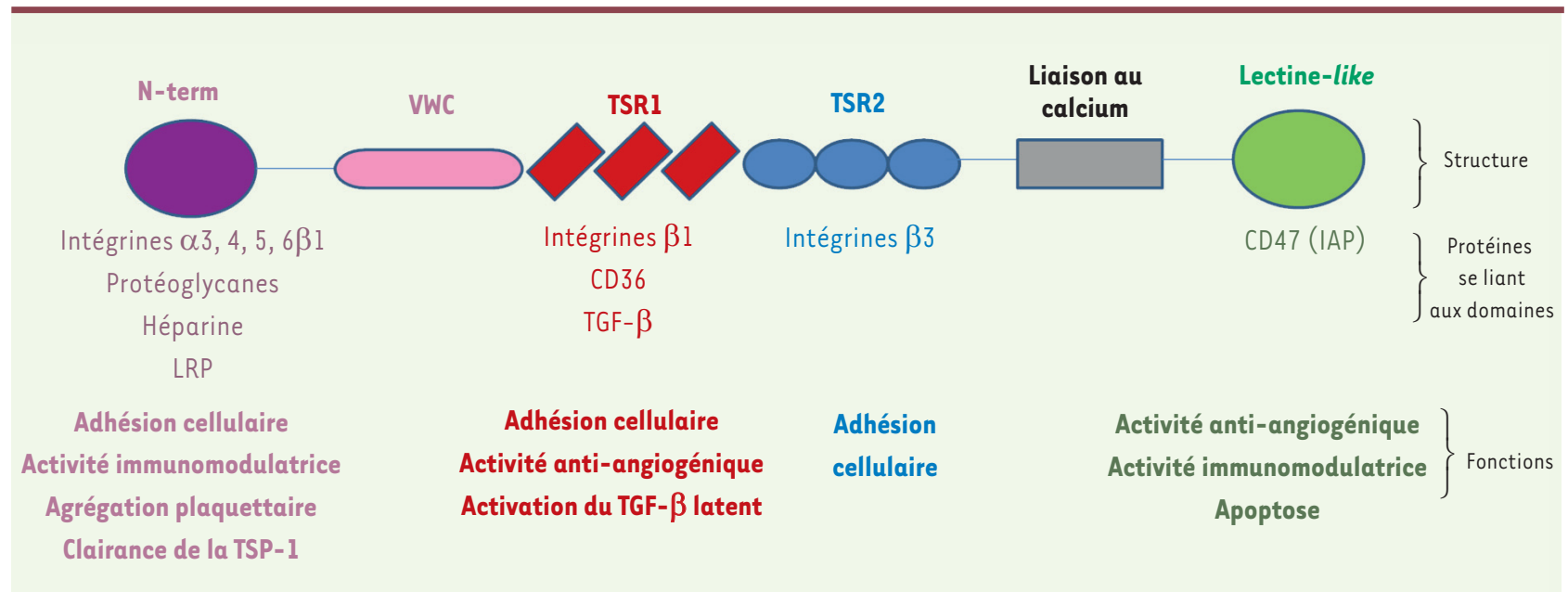

Figure 1. Structure, partenaires et activités de la thrombospondine-1. N-term : domaine amino-terminal ; VWC : domaine de type Von Willebrand; TSR1 : domaine de répétition de type 1 ; TSR2 : domaine de répétition de type 2. La figure est adaptée de [1, 2].

le TGF- $\beta$ mature et le LAP (latency associated peptide) via deux motifs situés dans le domaine TSR1. Les motifs WXXW de la TSP-1 fixent le TGF- $\beta$ latent, et le motif KRFK lie la séquence LSKL du LAP, libérant ainsi le TGF- $\beta$ mature qui pourra se fixer sur ses récepteurs [3].

\section{Activité anti-angiogénique}

En 1990, Good et al. rapportent pour la première fois le rôle antiangiogénique de la TSP-1 [4]. Celle-ci inhibe in vitro la migration de cellules endothéliales et in vivo la néovascularisation cornéenne stimulée par le basic fibroblast growth factor (bFGF) et le vascular endothelial growth factor (VEGF) [4]. Le phénotype vasculaire des souris TSP-l knock-out (KO) est caractérisé par une augmentation de la densité vasculaire rétinienne et musculaire, de la concentration musculaire en VEGF et de la capacité musculaire à l'effort [5]. La transfection de cellules cancéreuses par la séquence activatrice du TGF- $\beta$ induit une réduction de la croissance tumorale et une diminution du nombre et de la taille des vaisseaux. L'injection du peptide LSKL, qui empêche l'activation du TGF- $\beta$ par la TSP-1, restaure la croissance tumorale [6]. La TSP-1 peut bloquer la progression du cycle cellulaire et favoriser l'apoptose des cellules endothéliales en stimulant l'expression de Bax (Bcl-2-associated X protein) et en réduisant celle de Bcl-2 (B-cell lymphoma 2) [6]. La triple interaction TSP-1/ intégrines $\beta 1 / C D 36$ inhibe la migration des cellules endothéliales [6]. L'injection de peptides qui miment la séquence de la TSP-1 et se fixent au CD36 (ABT-510 et ABT-526) permet une diminution, voire une rémission tumorale chez l'animal, en induisant l'apoptose des cellules endothéliales et tumorales [6].

La TSP-1 empêche la libération du VEGF à partir de la matrice extracellulaire (MEC) et s'oppose ainsi aux effets pro-angiogéniques de ce dernier, en limitant l'activité de métalloprotéases, et elle agit comme inhibiteur compétitif du VEGF en se fixant, par son domaine amino-terminal, aux protéoglycanes des cellules endothéliales [7]. Grâce à son interaction avec CD36, les intégrines $\beta 1$ [8] et CD47 ${ }^{1}$ [9], la TSP-1 inhibe la phosphorylation du récepteur VEGF-R2, bloquant ainsi la voie de signalisation du VEGF.

L'interaction de la TSP-1 avec CD47 à la surface des cellules progénitrices endothéliales humaines limite leur prolifération et la formation de néovaisseaux [10]. Dans un modèle d'hypertension artérielle, le nombre de cellules endothéliales progénitrices circulantes est inversement proportionnel au taux de TSP-1 (Figure 2) [11]. L'inhibition de la voie du monoxyde d'azote (NO) joue un rôle déterminant dans l'effet anti-angiogénique de la TSP-1 (Figure 2). Cette dernière inhibe la production de NO induite par le VEGF et s'oppose à l'activation de sa voie de signalisation en limitant la production de GMP cyclique (GMPc) et l'activité de la protéine kinase dépendante du GMPc (cGKI). En bloquant la phosphorylation des chaînes de myosine par la cGKI, elle inhibe la relaxation des cellules musculaires lisses induite par le NO [12], et empêche ainsi l'adaptation de la perfusion tissulaire à l'ischémie. Le déficit en TSP-1 limite les lésions tissulaires et améliore la survie des animaux chez lesquels une ischémie aiguë cutanée, hépatique [12], cérébrale [13] ou d'un membre inférieur [12, 14] a été induite ; l'inhibition du CD47 ou son déficit génétique ont le même effet $[12,13]$. CD47 inhibe la voie du NO pour des concentrations 100 fois plus faibles de TSP-1 que celles nécessaires au CD36. En l'absence de CD47, mais pas de CD36, il n'y a pas d'inhibition de la voie du NO par la TSP-1, suggérant un rôle critique de CD47 [12].

\footnotetext{
${ }^{1}$ CD47 est une molécule de la famille des Ig qui se lie aux intégrines (IAP) et à la TSP-1. Elle est impliquée dans de nombreux processus cellulaires, et également comme un inhibiteur de la phagocytose par sa liaison à SIRP $\alpha$.
} 


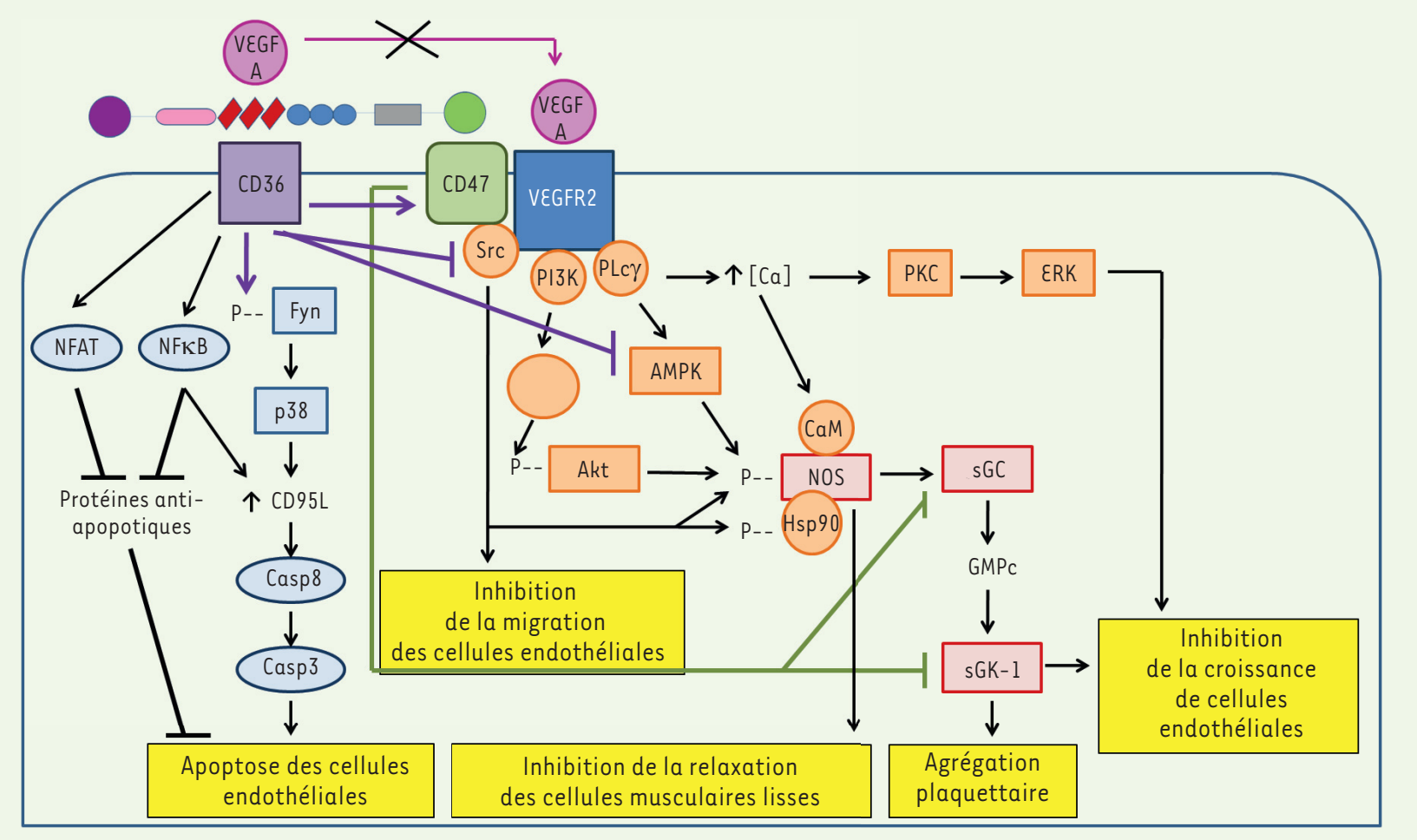

Figure 2. Activité anti-angiogénique de la thrombospondine-1. PLC $\gamma$ : phospholipase $C \gamma$; ERK : extracellular signal-regulated kinase ; Akt : protéine kinase B; Hsp90 : heat shock protein 90-kDa ; CaM : calmoduline. La figure est adaptée de [12].

Le rôle anti-angiogénique de la TSP-l dans les pathologies humaines non tumorales a été suggéré par des travaux sur l'artérite oblitérante des membres inférieurs. Le taux plasmatique de TSP-l est plus élevé chez les patients souffrant d'artérite que chez les témoins, et son expression est augmentée dans les tissus ischémiques et corrélée à la diminution de la densité capillaire [10]

\section{Activité immunomodulatrice}

La description initiale du phénotype des souris déficientes en TSP-1 rapportait une augmentation du nombre de leucocytes circulants et une inflammation pulmonaire mimant, à un moindre degré, celle observée chez les souris déficientes en TGF- $\beta$, suggérant un effet anti-inflammatoire de la TSP-l via l'activation du TGF- $\beta[3]$. Les résultats variables des études ultérieures menées in vitro ont souligné la complexité des effets immunologiques de la TSP-1 (Figure 3).

La fixation de TSP-1 aux récepteurs CD47 et CD36 [15] des cellules présentatrices d'antigènes conférerait à ces dernières un phénotype tolérogène en diminuant leur sécrétion de TNF $\alpha$ (tumor necrosis factor- $\alpha$ ) et d'interleukine 12 (IL12) [15]. Sa fixation au CD47 présent à la surface des lymphocytes T CD4 $4^{+}$naïfs ou mémoires induirait leur différenciation en lymphocytes $T$ régulateurs [16] et leur apoptose par l'intermédiaire de la protéine BNIP3 ( $\mathrm{BCl}-2$ ) adenovirus ElB 19-kDa-interacting protein 3) [17].
Les résultats d'autres études sont contradictoires. L'interaction de la TSP- 1 avec les intégrines $\alpha 4 \beta 1$ limiterait l'adhésion des lymphocytes T activés à VCAM-1 (vascular cell adhesion molecule-1), mais stimulerait leur production de MMP2 (matrix metalloproteinase 2) et -9 , favorisant leur pouvoir d'invasion tissulaire [18]. L'interaction entre les trois molécules, CD36 présent sur les cellules présentatrices de l'antigène, CD47 exprimé par les lymphocytes T et la TSP-1 matricielle, favoriserait l'activation et l'expansion clonale des lymphocytes T issus de patients souffrant de polyarthrite rhumatoïde [19]. La liaison de la TSP-1 au CD36 exprimé par les macrophages favoriserait la sécrétion de I'IL6, une cytokine pro-inflammatoire, tandis qu'elle inhiberait celle de I'IL10, une cytokine anti-inflammatoire. Néanmoins, la sécrétion d'IL10 serait restaurée en présence de TGF- $\beta$ sécrété par les macrophages, conférant à ces derniers un rôle de tolérance [20].

La plupart des études réalisées in vivo retrouvent un effet pro-inflammatoire de la TSP-1. Au cours de l'ischémiereperfusion, le déficit ou le blocage de la TSP-1, du CD36 ou du CD47 s'accompagne d'une protection tissulaire et d'une diminution de l'infiltrat inflammatoire, quel que soit l'organe lésé : la peau, le foie [12] ou le muscle 


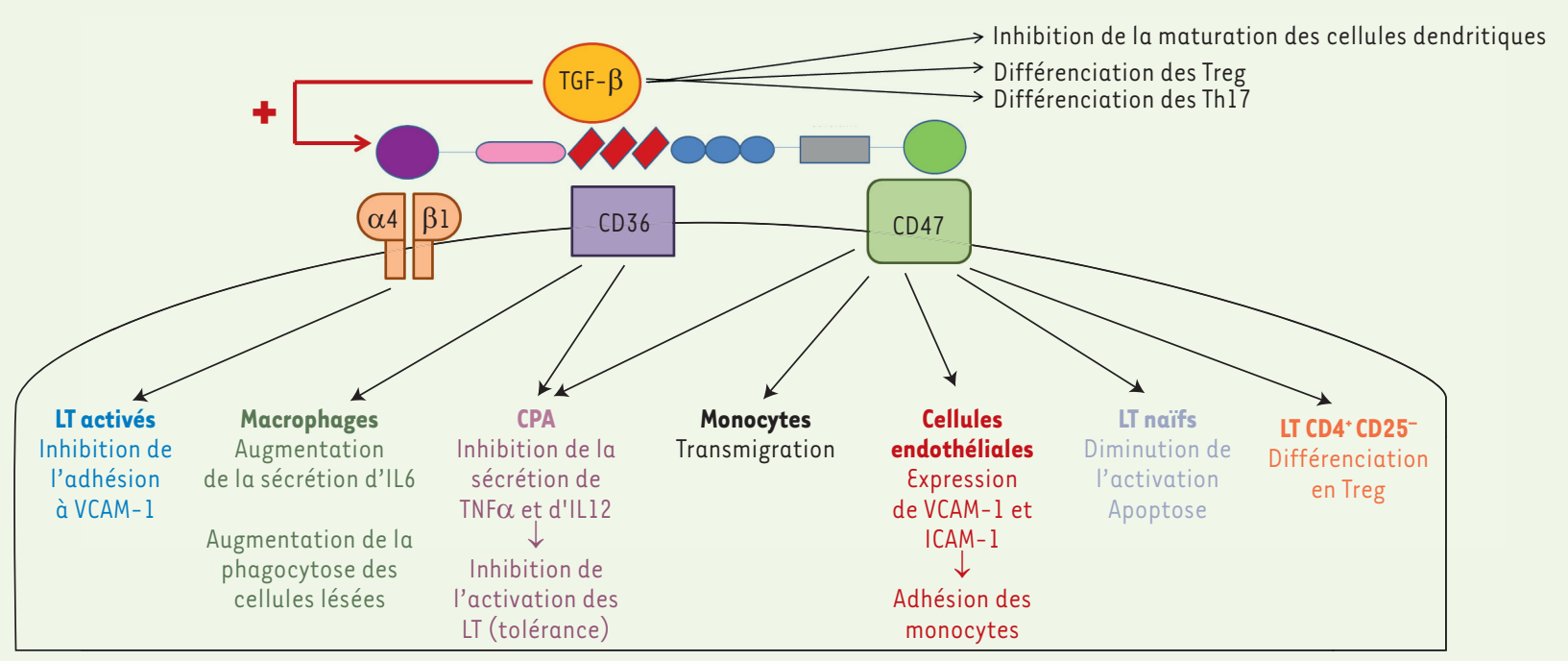

Figure 3. Activité immunomodulatrice de la thrombospondine-1 (études in vitro). CPA : cellules présentatrices d'antigène; LT : lymphocytes T.

[14]. Dans un modèle d'ischémie de la patte, le déficit en TSP-1 induit une augmentation de la sécrétion de cytokines anti-inflammatoires aux dépens des cytokines pro-inflammatoires, et une diminution de l'activité phagocytaire des macrophages [14]. Les souris déficientes en TSP-1 ont un retard de cicatrisation cutanée et une réduction de l'expression du facteur chimiotactique MCP-1 et de l'infiltration macrophagique [21]. Dans un modèle d'encéphalite auto-immune liée à une infiltration cérébrale par les lymphocytes Th17, I'administration du peptide LSKL, bloquant l'activation du TGF- $\beta$ par la TSP-1, retarde la survenue des symptômes neurologiques [22]. Chez les souris déficientes en TSP-1, cette encéphalite est atténuée grâce à une réduction de la sécrétion d'IL17 et d'interféron- $\gamma($ IFN- $\gamma)$. Ce bénéfice est annulé par la restauration de l'activation du TGF- $\beta$ [23].

La TSP-1 interviendrait aussi dans la régulation de l'immunité innée. Elle favoriserait l'adhésion des monocytes à l'endothélium en induisant l'expression des molécules VCAM-l et ICAM-1 (intercellular adhesion molecule-1) [24]. Cependant, au cours du sepsis bactérien ou fongique, la mortalité des souris TSP-l est réduite et associée à une diminution de la libération de cytokines pro-inflammatoires, du recrutement des leucocytes [25], et de l'activité phagocytaire [25, 26].

\section{Expression de la thrombospondine-1 au cours des néphropathies expérimentales}

Chez l'adulte sain, l'expression de la TSP-l au sein du parenchyme rénal est très faible et se limite à la capsule de Bowman et à l'interstitium. En conditions pathologiques, elle a été analysée in vivo dans différents modèles de néphropathies. Après ischémie-reperfusion [27], réduction néphronique [28], obstruction urétérale unilatérale [29], mais aussi au cours du vieillissement rénal [30], du diabète [31] et de nombreuses autres néphropathies glomérulaires expérimentales [32, 33], les cellules épithéliales tubulaires expriment de novo la TSP-1. In vitro, I'hypoxie [34] et l'enrichissement du milieu de culture en glucose [31, 35] stimulent la production de TSP-l par les cellules épithéliales tubulaires. Au cours de ces différents modèles de néphropathie, on note également une expression interstitielle de TSP-1 corrélée à l'extension de la fibrose. Dans trois modèles de glomérulopathie, Hugo et al. ont montré que les myofibroblastes et quelques macrophages interstitiels expriment la TSP-1 [33]. Bien que les lymphocytes soient une source connue de TSP-l, aucune étude n'a, à notre connaissance, décrit l'expression de TSP-1 par les lymphocytes localisés dans le parenchyme rénal.

Tous les modèles de glomérulopathies mettent en évidence une expression glomérulaire de TSP-1 dont la topographie correspond aux cellules mésangiales. In vitro, l'expression de la TSP-1 par les cellules mésangiales est stimulée par l'angiotensine II [36], le bFGF, le PDGF (plateled derived growth factor) [37] et l'enrichissement du milieu de culture en glucose [31]. Enfin, le glucose peut induire in vitro la transcription du gène tspl par les podocytes [38].

Bien que la présence de TSP- 1 dans des cellules endothéliales isolées de reins de souris ait été détectée par Western blot [39], aucune des données immunohistochimiques publiées n'a mis en évidence d'expression endothéliale de TSP-l dans le parenchyme rénal, à notre connaissance.

\section{Rôle de la thrombospondine-1 dans le développement des néphropathies expérimentales}

\section{Par l'activation du TGF- $\beta$}

En 1998, Hugo et al. ont décrit, dans trois modèles de glomérulonéphrite chez le rat (induite par les anticorps 
anti-Thyl et la puromycine, et dans la néphrite de Heymann), une expression corticale de novo de l'ARNm de $t s p-1$ et de la protéine TSP-1 par les cellules tubulaires, les macrophages et surtout les myofibroblastes, superposable à celle du TGF- $\beta$. Elle précède la survenue de la fibrose tubulo-interstitielle, et est corrélée à son extension [33]. Chez le rat, l'obstruction urétérale unilatérale induit une expression de novo de l'ARNm de tsp-l et de la protéine TSP-l par les tubules lésés. Cette expression est corrélée au contenu rénal en TGF- $\beta$, et diminue parallèlement à celle du TGF- $\beta$ après désobstruction urétérale [29].

L'inhibition spécifique de l'activation du TGF- $\beta$ par la TSP-1 (via l'administration du peptide LSKL) a été étudiée dans trois modèles de néphropathie. La perfusion de ce peptide, après induction d'une glomérulonéphrite membrano-proliférative chez le rat, réduit l'accumulation de MEC dans les glomérules et le débit de protéinurie [40]. L'injection de LSKL à des souris diabétiques, après une néphrectomie unilatérale, réduit le taux urinaire de TGF- $\beta$ actif, l'expression rénale des protéines Smad2 (mothers against decapentaplegic homolog 2) et -3 phosphorylées, l'accumulation interstitielle de fibronectine, la perte d'expression de la néphrine par les podocytes et l'albuminurie [41]. Enfin, l'injection de LSKL après obstruction urétérale unilatérale diminue l'expression rénale de Smad2 phosphorylée, les lésions tubulaires, la fibrose interstitielle, et s'associe à une réduction de l'expression de l'ARNm de tsp-l et de sa protéine, suggérant un rétrocontrôle positif du TGF- $\beta$ actif sur l'expression de la TSP-1 [42].

L'injection intrarénale d'oligonucléotides antisens anti-TSP-1 permet une réduction de l'activation glomérulaire du TGF- $\beta$ et de l'accumulation de protéines de la MEC (collagènes I et IV, fibronectine) dans un modèle de glomérulonéphrite membrano-proliférative induite par des anticorps antiThyl chez le rat [43]. Après induction d'un diabète par la streptozocine chez les souris TSP-1 K0, l'expression glomérulaire de TGF- $\beta$, des Smad2 et -3 phosphorylées et de PAI-1 (plasminogen activator inhibitor-1), l'accumulation mésangiale de fibronectine et de collagène IV, les lésions podocytaires, l'infiltration interstitielle par les macrophages et les lymphocytes, et la protéinurie sont réduites [44]. Enfin, l'activation rénale du TGF- $\beta$ et les lésions tubulo-interstitielles induites par l'obstruction urétérale unilatérale sont atténuées chez la souris TSP-1 KO [29].

\section{Par son activité anti-angiogénique}

En 2001, Kang et al. ont montré que la raréfaction des capillaires et la diminution de la prolifération des cellules endothéliales glomérulaires et tubulaires observées au cours du vieillissement [30] ou après réduction néphronique [28] chez le rat, sont corrélées positivement à l'expression de TSP-1, et négativement à celle du VEGF.

En 2005, Thakar et al. ont identifié par PCR (polymerase chain reaction) micro-array le gène codant pour la TSP-1 comme l'un des deux gènes les plus exprimés dans des reins de rat après ischémie-reperfusion. L'expression de la TSP-1 est maximale trois heures après reperfusion, puis décroît au cours du temps pour se normaliser à 48 heures. Elle est essentiellement exprimée par les cellules tubulaires proximales lésées dont elle induit l'apoptose selon un processus dépendant des caspases. Enfin, les souris TSP-1 KO sont protégées contre les lésions tubulaires et l'insuffisance rénale aiguë induites par l'ischémie-reperfusion [27].
Notre équipe a également observé cette protection tubulaire chez les souris TSPI-KO après obstruction urétérale unilatérale. L'obstruction urétérale unilatérale entraîne une atrophie corticale, des dilatations tubulaires, une raréfaction des capillaires péritubulaires, ainsi qu'une fibrose et une inflammation interstitielles importantes. Ces anomalies sont corrélées à l'expression interstitielle et tubulaire de la TSP-1. Après désobstruction, l'expression de la TSP-1 décroît avec la régression des lésions. Les souris déficientes en TSP-l présentent des lésions tubulaires, une perte d'expression de la mégaline et une atrophie corticale réduites par rapport aux animaux sauvages. Cette protection s'associe à une expression accrue du VEGF et de son récepteur VEGF-R2, et à une préservation du réseau capillaire péritubulaire [29].

\section{Par son activité pro-inflammatoire}

La responsabilité de l'activité pro-inflammatoire de la TSP-l dans le développement des néphropathies a été suggérée pour la première fois en 2001. Kang et al. ont montré une relation linéaire entre l'expression tubulaire et glomérulaire de la TSP-1 et l'infiltration macrophagique après néphrectomie des cinq sixièmes (néphrectomie unilatérale et ablation des deux tiers du rein controlatéral) chez le rat [28].

Trois études, toutes trois réalisées chez des souris TSP-1 $K O$, ont démontré l'effet pro-inflammatoire de la TSP-1 au cours de néphropathies expérimentales. Après induction d'un diabète par la streptozocine, les souris TSP-1 KO présentent une glomérulosclérose, une fibrose interstitielle et un infiltrat interstitiel par les macrophages et les lymphocytes moins marqués que les animaux sauvages [44]. Trois semaines après l'injection d'anticorps antimembrane basale glomérulaire, il y a atténuation des lésions de glomérulonéphrite extracapillaire (nombre de croissants extracapillaires et de dépôts de fibrine, infiltration interstitielle lymphocytaire et macrophagique) et du dysfonctionnement rénal chez les souris déficientes en TSP-1 [32]. Enfin, après obstruction urétérale unilatérale, l'infiltrat lymphocytaire interstitiel et l'expression des ARNm de I'IL17 et du facteur de transcription ROR $\gamma$ T, associés à l'activation de la voie Th17 et de MCP-1, sont réduits chez les animaux $\mathrm{KO}$ [29].

\section{Thrombospondine- 1 et pathologie rénale chez l'homme}

En 1994, Mc Gregor et al. ont étudié pour la première fois chez l'homme l'expression des thrombospondines sur des biopsies rénales humaines. Dans le rein normal, l'expression des TSP est très faible et limitée à l'interstitium. En revanche, elle est intense dans les synéchies 
floculo-capsulaires et l'interstitium au cours de la hyalinose segmentaire et focale, et au sein des croissants cellulaires des glomérulonéphrites extracapillaires, tandis que les croissants fibreux sont peu marqués [45]. Au cours de la néphropathie diabétique, l'expression mésangiale (notamment au sein des nodules de Kimmelstiel-Wilson), podocytaire, tubulaire et interstitielle de la TSP-1 augmente avec la sévérité des lésions histologiques [31]. Elle s'accompagne d'une augmentation de l'expression du CTGF (connective tissue growth factor), du TGF- $\beta$ et des protéines Smad2 et -3 phosphorylées intervenant dans la voie de signalisation du TGF- $\beta$ activé.

\section{Conclusion}

Après une agression rénale aiguë ou chronique, la TSP-1 contribue au développement des lésions parenchymateuses par au moins trois mécanismes: I'activation du TGF- $\beta$, un effet pro-inflammatoire et un effet anti-angiogénique. Son inhibition ou celle de ses partenaires, notamment le récepteur CD47, par des anticorps bloquant ou des analogues compétitifs, pourrait constituer une nouvelle cible thérapeutique dans la prise en charge des maladies rénales chroniques. $\diamond$

\section{SUMMARY}

Role of thrombospondin- 1 in the development of kidney diseases Thrombospondin-1 (TSP-1) is a 450-kDa matricellar glycoprotein. By its various domains, it can interact with various partners and exhibit antiangiogenic, pro-apoptotic and immunomodulatory activities. TSP-1 is also a major endogenous activator of the pro-fibrotic growth factor TGF$\beta$. In healthy adult renal parenchyma, TSP-1 expression is very scarce and limited to Bowman's capsule and interstitium. During nephropathies, many cell types can express or secrete TSP-1 (mesangial, endothelial, smooth muscle, tubular cells, podocytes and fibroblasts) depending on the nature of injury and the evolutive stage of the disease. Inhibition of the different domains of TSP-1 using specific antibodies or peptides, blockade of TSP-1 expression by antisense oligonucleotides and use of knock-out mice, allowed to identify the role of TSP-1 in various models of experimental nephropathy. All these studies demonstrated a deleterious effect of TSP- 1 on renal repair by inducing TGF- $\beta$ and fibrosis, decreasing VEGF and capillary density, and enhancing inflammatory cells recruitment. Thus, TSP-1 represents a potential therapeutic target for the management of chronic kidney diseases. $\diamond$

\section{REMERCIEMENTS}

Naïke Bigé et Fanny Lepeytre remercient respectivement la Fondation pour la recherche médicale et l'Assistance publique-hôpitaux de Paris pour leur soutien financier.

\section{LIENS D'INTÉRÊT}

J.J. Boffa déclare participer ou avoir participé à des interventions ponctuelles (essais cliniques, travaux scientifiques, rapports d'expertise, conseil, conférences, colloques) pour les entreprises Amgen, Novartis, Roche Guerbet, Fresenius.

$N$. Bigé, F. Lepeytre et N. Shweke déclarent n'avoir aucun lien d'intérêt concernant les données publiées dans cet article.

\section{RÉFÉRENCES}

Une annexe contenant 150 références bibliographiques (Annexe 1) est également à consulter sur le site de médecine/sciences, www. medecinesciences.org à cette URL http://www.medecinesciences. org/10.1051/medsci/20132912017/olm

1. Carlson CB, Lawler J, Mosher DF. Structures of thrombospondins. Cell Mol Life Sci $2008 ; 65: 672-86$.

2. Lawler J. The functions of thrombospondin-1 and-2. Curr Opin Cell Biol $2000 ; 12: 634-40$.

3. Crawford SE, Stellmach V, Murphy-Ullrich JE, et al. Thrombospondin-1 is a major activator of TGF-betal in vivo. Cell $1998 ; 93$ : 1159-70.

4. Good DJ, Polverini PJ, Rastinejad F, et al. A tumor suppressor-dependent inhibitor of angiogenesis is immunologically and functionally indistinguishable from a fragment of thrombospondin. Proc Natl Acad Sci USA $1990 ; 87: 6624-8$.

5. Malek MH, Olfert IM. Global deletion of thrombospondin-1 increases cardiac and skeletal muscle capillarity and exercise capacity in mice. Exp Physiol $2009 ; 94: 749-60$.

6. Mirochnik Y, Kwiatek A, Volpert OV. Thrombospondin and apoptosis: molecular mechanisms and use for design of complementation treatments. Curr Drug Targets $2008 ; 9: 851-62$.

7. Rodriguez-Manzaneque JC, Lane TF, Ortega MA, et al. Thrombospondin-1 suppresses spontaneous tumor growth and inhibits activation of matrix metalloproteinase- 9 and mobilization of vascular endothelial growth factor. Proc Natl Acad Sci USA 2001 ; 98 : 12485-90.

8. Zhang X, Kazerounian S, Duquette M, et al. Thrombospondin-1 modulates vascular endothelial growth factor activity at the receptor level. Faseb J $2009 ; 23: 3368-76$.

9. Kaur S, Martin-Manso G, Pendrak ML, et al. Thrombospondin-1 inhibits VEGF receptor-2 signaling by disrupting its association with CD47. J Biol Chem $2010 ; 285: 38923-32$.

10. Smadja DM, d'Audigier C, Bieche I, et al. Thrombospondin-1 is a plasmatic marker of peripheral arterial disease that modulates endothelial progenitor cell angiogenic properties. Arterioscler Thromb Vasc Biol 2011 ; 31 : 551-9.

11. Xie HH, Zhou S, Chen DD, et al. GTP cyclohydrolase I/BH4 pathway protects EPCs via suppressing oxidative stress and thrombospondin- 1 in saltsensitive hypertension. Hypertension $2010 ; 56$ : 1137-44.

12. Isenberg JS, Martin-Manso G, Maxhimer JB, Roberts DD. Regulation of nitric oxide signalling by thrombospondin 1: implications for anti-angiogenic therapies. Nat Rev Cancer 2009 ; 9 : 182-94.

13. Jin G, Tsuji K, Xing C, et al. CD47 gene knockout protects against transient focal cerebral ischemia in mice. Exp Neurol 2009 ; 217 : 165-70.

14. Brechot N, Gomez $\varepsilon$, Bignon M, et al. Modulation of macrophage activation state protects tissue from necrosis during critical limb ischemia in thrombospondin-1-deficient mice. PloS One 2008 ; 3 : e3950.

15. Masli S, Turpie B, Streilein JW. Thrombospondin orchestrates the tolerancepromoting properties of TGFbeta-treated antigen-presenting cells. Int Immunol $2006 ; 18: 689-99$.

16. Grimbert P, Bouguermouh S, Baba N, et al. Thrombospondin/CD47 interaction: a pathway to generate regulatory $T$ cells from human CD4 CD25- T cells in response to inflammation. J Immunol 2006 ; 177 : 3534-41.

17. Lamy L, Foussat A, Brown $\varepsilon$ J, et al. Interactions between CD47 and thrombospondin reduce inflammation. J Immunol 2007 ; 178 : 5930 -9.

18. Li Z, Calzada MJ, Sipes JM, et al. Interactions of thrombospondins with alpha4betal integrin and CD47 differentially modulate T cell behavior.J Cell Biol $2002 ; 157$ : 509-19.

19. Vallejo AN, Mugge LO, Klimiuk PA, et al. Central role of thrombospondin-1 in the activation and clonal expansion of inflammatory T cells. J Immunol 2000 ; 164 : 2947-54.

20. Yamauchi $Y$, Kuroki M, Imakiire T, et al. Thrombospondin-1 differentially regulates release of IL-6 and IL-10 by human monocytic cell line U937. Biochem Biophys Res Commun 2002 ; 290 : 1551-7.

21. Agah A, Kyriakides TR, Lawler J, Bornstein P. The lack of thrombospondin-1 (TSP1) dictates the course of wound healing in double-TSP1/TSP2-null mice. Am J Pathol 2002 ; $161: 831-9$.

22. Lanz TV, Ding Z, Ho PP, et al. Angiotensin II sustains brain inflammation in mice via TGF-beta. J Clin Invest 2010 ; 120 : 2782-94.

23. Yang K, Vega JL, Hadzipasic M, et al. Deficiency of thrombospondin-1 reduces Th17 differentiation and attenuates experimental autoimmune encephalomyelitis. J Autoimmun 2009 ; 32 : 94-103. 


\section{RÉFÉRENCES}

24. Narizhneva NV, Razorenova OV, Podrez EA, et al. Thrombospondin-1 up-regulates expression of cel adhesion molecules and promotes monocyte binding to endothelium. Faseb J 2005 ; 19 : 1158-60.

25. Martin-Manso G, Navarathna DH, Galli S, et al. Endogenous thrombospondin-1 regulates leukocyte recruitment and activation and accelerates death from systemic candidiasis. Plos One $2012 ; 7:$ e 48775 .

26. McMaken S, Exline MC, Mehta P, et al. Thrombospondin-l contributes to mortality in murine sepsis through effects on innate immunity. PloS One 2011 ; 6 : e19654.

27. Thakar CV, Zahedi K, Revelo MP, et al. Identification of thrombospondin I (TSP-1) as a novel mediator of cell injury in kidney ischemia. J Clin Invest $2005 ; 115$ : 3451-9.

28. Kang DH, Joly AH, Oh SW, et al. Impaired angiogenesis in the remnant kidney model: I. Potential role of vascular endothelial growth factor and thrombospondin-1.J Am Soc Nephrol 2001 ; 12 : 1434-47.

29. Bige N, Shweke N, Benhassine S, et al. Thrombospondin-1 plays a profibrotic and proinflammatory role during ureteric obstruction. Kidney Int 2012 ; 81 : 1226-38.

30. Kang DH, Anderson S, Kim YG, et al. Impaired angiogenesis in the aging kidney: vascular endothelial growth factor and thrombospondin-1 in renal disease. Am J Kidney Dis 2001 ; 37 : 601-11.

31. Wahab NA, Schaefer L, Weston BS, et al. Glomerular expression of thrombospondin-1, transforming growth factor beta and connective tissue growth factor at different stages of diabetic nephropathy and their interdependent roles in mesangial response to diabetic stimuli. Diabetologia $2005 ; 48: 2650-60$.

32. Hochegger K, Knight S, Hugo C, et al. Role of thrombospondin-1 in the autologous phase of an accelerated model of anti-glomerular basement membrane glomerulonephritis. Nephron 2004 ; $96:$ e31-8.

33. Hugo C, Shankland SJ, Pichler RH, et al. Thrombospondin 1 precedes and predicts the development of tubulointerstitial fibrosis in glomerular disease in the rat. Kidney Int $1998 ; 53: 302-11$.

34. Rogers NM, Thomson AW, Isenberg JS. Activation of parenchymal CD47 promotes renal ischemiareperfusion injury. J Am Soc Nephrol 2012 ; 23 : 1538-50.

35. Yung $\mathrm{S}$, Lee $\mathrm{CY}$, Zhang $\mathrm{Q}$, et al. Elevated glucose induction of thrombospondin-1 up-regulates fibronectin synthesis in proximal renal tubular epithelial cells through TGF-betal dependent and TGF-betal independent pathways. Nephrol Dial Transplant 2006 ; 21 : 1504-13.
36. Naito T, Masaki T, Nikolic-Paterson DJ, et al. Angiotensin II induces thrombospondin-1 production in human mesangial cells via p38 MAPK and JNK: a mechanism for activation of latent TGF-betal. Am J Physiol 2004 ; 286 : F278-87.

37. Hugo C, Pichler R, Meek R, et al. Thrombospondin $\mathrm{l}$ is expressed by proliferating mesangial cells and is up-regulated by PDGF and bFGF in vivo. Kidney Int 1995 ; 48 : 1846-56.

38. Han SH, Yang S, Jung DS, et al. Gene expression patterns in glucosestimulated podocytes. Biochem Biophys Res Commun 2008 ; 370 : 514-8.

39. Sheibani N, Morrison ME, Gurel Z, et al. BIM deficiency differentially impacts the function of kidney endothelial and epithelial cells through modulation of their local microenvironment. Am J Physiol 2012 ; 302 : F809-19.

40. Daniel C, Wiede J, Krutzsch HC, et al. Thrombospondin-1 is a major activator of TGF-beta in fibrotic renal disease in the rat in vivo. Kidney Int $2004 ; 65: 459-68$.

41. Lu A, Miao M, Schoeb TR, et al. Blockade of TSP1-dependent TGF-beta activity reduces renal injury and proteinuria in a murine model of diabetic nephropathy. Am J Pathol $2011 ; 178: 2573-86$.

42. Xie XS, Li FY, Liu HC, et al. LSKL, a peptide antagonist of thrombospondin-1, attenuates renal interstitial fibrosis in rats with unilateral ureteral obstruction. Arch Pharmacal Res $2010 ; 33: 275-84$.

43. Daniel C, Takabatake Y, Mizui M, et al. Antisense oligonucleotides against thrombospondin-1 inhibit activation of tgf-beta in fibrotic renal disease in the rat in vivo. Am J Pathol $2003 ; 163: 1185-92$.

44. Daniel C, Schaub K, Amann K, et al. Thrombospondin- 1 is an endogenous activator of TGF-beta in experimental diabetic nephropathy in vivo. Diabetes $2007 ; 56: 2982-9$

45. McGregor B, Colon S, Mutin M, et al. Thrombospondin in human glomerulopathies. A marker of inflammation and early fibrosis. Am J Pathol 1994 ; 144 : 1281-7.

\section{TIRÉS À PART}

J.J. Boffa

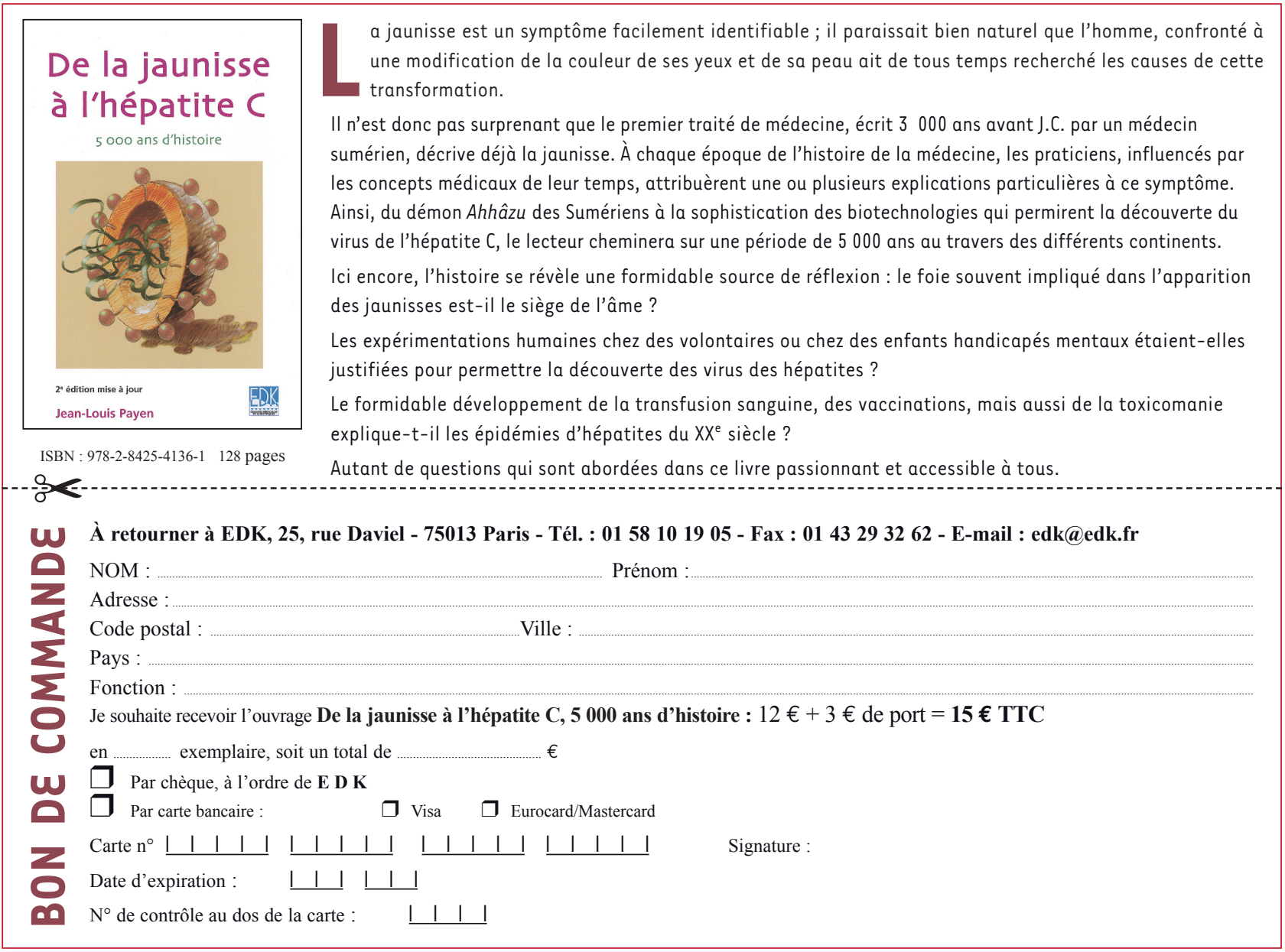

